\author{
Military Technical College \\ Kobry El-Kobbah, \\ Cairo, Egypt.
}

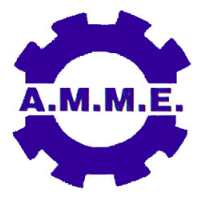

\title{
A PROPOSED METHOD FOR DETECTING THE CHAOTIC BEHAVIOUR IN DYNAMICAL SYSTEMS
}

\author{
A. A. Mohamed ${ }^{1}$ and S. H. Darwish ${ }^{2}$
}

\begin{abstract}
The important step in studying the qualitative behavior of non-linear dynamical system is how to detect the presence of chaos. There are several methods that used to determine the presence of chaos signature. This paper presents a proposed method in detecting the presence of chaos. This method combines two techniques namely: the normal form analysis and largest Lyapunov exponent (LLE). Computer programs were generated to investigate these two techniques and the proposed detecting method. An example was given to furnish the herein given computer algebra techniques based on real applications. The obtained results in this work were verified with that published by other researchers. The proposed method can provide highly active and efficient ability when studying the nature of non-linear dynamical systems and its chaotic presence.
\end{abstract}

\section{KEY WORDS}

Chaos, Normal form, Largest Lyapunov exponent.

1 Faculty of Engineering, Pharos University, Alexandria, Egypt. Email: Azima65@hotmail.com.

2 Faculty of Engineering, Pharos University, Alexandria, Egypt. Email: Salord1960@yahoo.com. 


\section{INTRODUCTION}

Many tools can be used to study the qualitative behavior of linear or non-linear dynamical systems. In general, non-linear systems cannot be analyzed completely in a systematic manner [1]. Therefore, some approaches and techniques can be used to represent the non-linear system such as: the use of describing function approach can be used when a transfer function of the linear part of the non-linear system is available; the use of Volterra series [2] in case of weakly non-linear systems, or the use of harmonic balance when single non-linearity exists. Other numerical techniques with advantages and disadvantages to analyze non-linear systems were developed [3]. Many problems and limitations arise when using the above approaches and techniques. The biggest problem is how to find an algorithm that enables the solution of the non-linear dynamical system and keeps minimum errors; besides, it should also offer simplicity in use as well as quick convergence [4].

This paper focuses on two approaches for studying the qualitative behavior of the non-linear system. The first one is the normal form theory [5],[6],[7],[8] and the second is the LLE technique [11]. The normal forms are used to facilitate and help in diagnosing the onset of chaos in non-linear systems while calculations of Lyapunov exponents are a way to study where a system is chaotic or not. In the speaking about Lyapunov exponents, the largest one is meant. A positive LLE indicates that the system is chaotic, while a negative one indicates the non-chaotic behavior. The LLE can also be used to analyze the stability of non-linear systems [12],[13],[14]. A proposed algorithm was created to satisfy the idea of the suggested methodology.

In the following, the outline of the normal forms method for non-linear dynamical systems and the computation procedures for the LLE are presented. In addition, the proposed method for detecting the chaotic behavior in dynamical systems is illustrated and a comparative study for validating this method is presented.

\section{NORMAL FORM FOR NON-LINEAR DYNAMICAL SYSTEM}

Consider the autonomous non-linear system given by the following state equation [2]:

$$
\frac{d x(t)}{d t}=A x(t)+B F_{n}(x(t))
$$

where $A$ and $B$ are real matrices of constant coefficients and compatible dimensions, and $F_{n}(x(t))$ is the vector of non-linear functions that satisfy some regularity conditions such as smoothness, continuity, and should be totally Lipchitz.

The normal form theory depends upon successive coordinate transformations to construct a simple form and to find a new system with a topologically that conjugates to the original system in a simple canonical form. However there are many techniques for finding the normal forms and most of these techniques lack generality. Hartman-Grobman method uses the eigenvalues of the linearized system [5]. If one of the eigenvalues lies on the imaginary axis, the linearization fails, and the process is terminated. The Poincare' method does not work when there are resonance 
relation between the Eigenvalues [8.]. Both Poincare' and Taken methods give the same result for vector fields having a diagonalizable linear part [10]. Poincare' method not applied to non-diagonalizable vector fields. Ushiki normal form method which is the most suitable technique; is based on the Lie brackets and the K-jets [67-8].

Ushiki method may be considered as a refinement of Takens' method. The advantage of this method is that it does not need an eigenvalue decomposition technique. In what follows the adaptation of the Ushiki method in the calculation of the normal form of non-linear system described by equation (1) is presented. The $\mathrm{K}^{\text {th }}$ order normal form of the non-linear system in (1) can be obtained by solving the following equation [8]:

$$
\frac{d}{d t} g_{k}(t)=\pi_{k}\left(\left[Y^{k-1}, V^{k-1}+g_{k}(t)\right]_{k}\right)
$$

and

$$
\left[Y^{k-1}, V^{k-1}\right]^{k-1}=0
$$

where $\left[Y^{k-1}, V^{k-1}\right]^{k-1}$ is the $\mathrm{K}$-jet of $\left[Y^{k-1}, V^{k-1}\right]$ at $0, \pi_{K}$ is the natural projection of $\mathrm{H}_{\mathrm{k}}$ along $\mathrm{B}_{\mathrm{k}}$ by $\pi_{\mathrm{k}}: \mathrm{H}_{\mathrm{k}} \rightarrow \mathrm{G}_{\mathrm{k}}, V^{K} \in x_{0}^{K}, \mathrm{Y}_{K} \in H_{K},\left[Y^{k-1}, V^{k-1}\right]$ is the bracket of $Y^{k-1}$ and $V^{k-1}, x_{0}$ is the vector space of all smooth vector fields (i.e., $C^{\infty}$ ), $x_{0}^{K}$ is the vector space of all $K$-jets of the vector fields in $\chi_{0}$ at 0 (obtained by truncating all terms of degree greater than $\mathrm{k}), H_{K}$ is the subspace consisting of all vector fields described by a homogeneous polynomial of degree $K, H_{K} \subset x_{0}^{K}, B_{K}$ is the subspace of $\mathrm{H}_{\mathrm{k}}$ consisting of the image of the linear map, and $B_{K} \subset H_{K}, G_{K}$ is the complementary subspace to $B_{K}$.

\section{LYAPUNOV EXPONENTS}

There is dependency between Lyapunov exponents and the chaotic properties of dynamical systems. There are several techniques for finding the Lyapunov exponents of the non-linear systems given by equation (1) [11-16]. However, no single technique appears to be optimal for calculating the Lyapunov exponents. For calculating the LLE depending upon the time domain solution of the state equation, some methods can be described as:

\section{Standard Method}

This method is most popular method to compute the Lyapunov exponents that combines the numerical integration of the linear evaluation equation with the GramSchmidt reorthonormalization process applied periodically. To find the approximate exponents $\lambda_{i}(\mathrm{t})$ at time $\mathrm{t}=\mathrm{T}$, the renormalization internal T was picked, so that $\mathrm{T}=$ $r T$ with $r \in N$, the linear evaluation equation is integrated over T and the GramSchmidt process applied. This is repeated a total $r$ times keeping the norms from the Gram-Schmidet (GS). The GS ensures that the direction and rates of growth are measured correctly. The Lyapunov exponents are [12]: 


$$
\lambda_{i}=\lim _{r \rightarrow \infty} \frac{\sum_{m=1}^{r} \log \left(N_{i}(m)\right)}{r T}
$$

where $\mathrm{Ni}(m)$ is the norm of the distance between two trajectory points, $r$ is the number of iterations, and $T$ is the interval period of renormalization.

This method needs a good choice of $T$ and large number of iterations since a bad choice of $T$ may cause the algorithm to fail.

\section{Q-R Algorithm}

It is another technique for finding the Lyapunov exponents. This method depends upon decomposition of the fundamental solution of the system. The fundamental solution is expressed as $M(t)=Q(t) R(t)$, where $Q(t)$ is an orthogonal matrix and $R(t)$ is an upper triangular matrix which are functions of both $Q(t)$ and the Jacobian matrix of the system (1) [11-15]. The Lyapunov exponents are obtained from the diagonal elements of the matrix $R(t)$ and are given by [15]:

$$
\lambda_{i}=\lim _{t \rightarrow \infty} \frac{1}{t} \log R_{i i}(t), 1 \leq i \leq n
$$

This method needs accurate evaluation of the orthogonal matrix, since the error in orthogonality leads to a break down in the computations of Lyapunov exponents due to numerical over and under flows. The two methods require rescaling, reorthogonalization and large number of iterations.

A proposed algorithm based on combining the normal form theory and the numerical calculation of the LLE to satisfy the qualitative behavior of non-linear systems is illustrated in the next section.

\section{PROPOSED METHOD FOR DETECTING THE CHAOTIC OF A DYNAMICAL SYSTEM}

This method don't need rescaling or re-orthogonalization so it lends itself to fast computation, easy implementation and reliable for all systems of higher order. It also avoids numerical over flow and takes the advantage of making use of all available data in the time series solution of the differential equation. Figure 1 illustrates the proposed algorithm procedures as follows: the normal form expressions obtained from the system of differential equations are used to convert the non-linear dynamical system to simple equivalent one. Time series data were generated from the equivalent system to evaluate LLE. Then the presence of chaos can be detected.

\section{The proposed approach proceeds as follows:-}

- Obtain the normal form expressions as given in (2). 


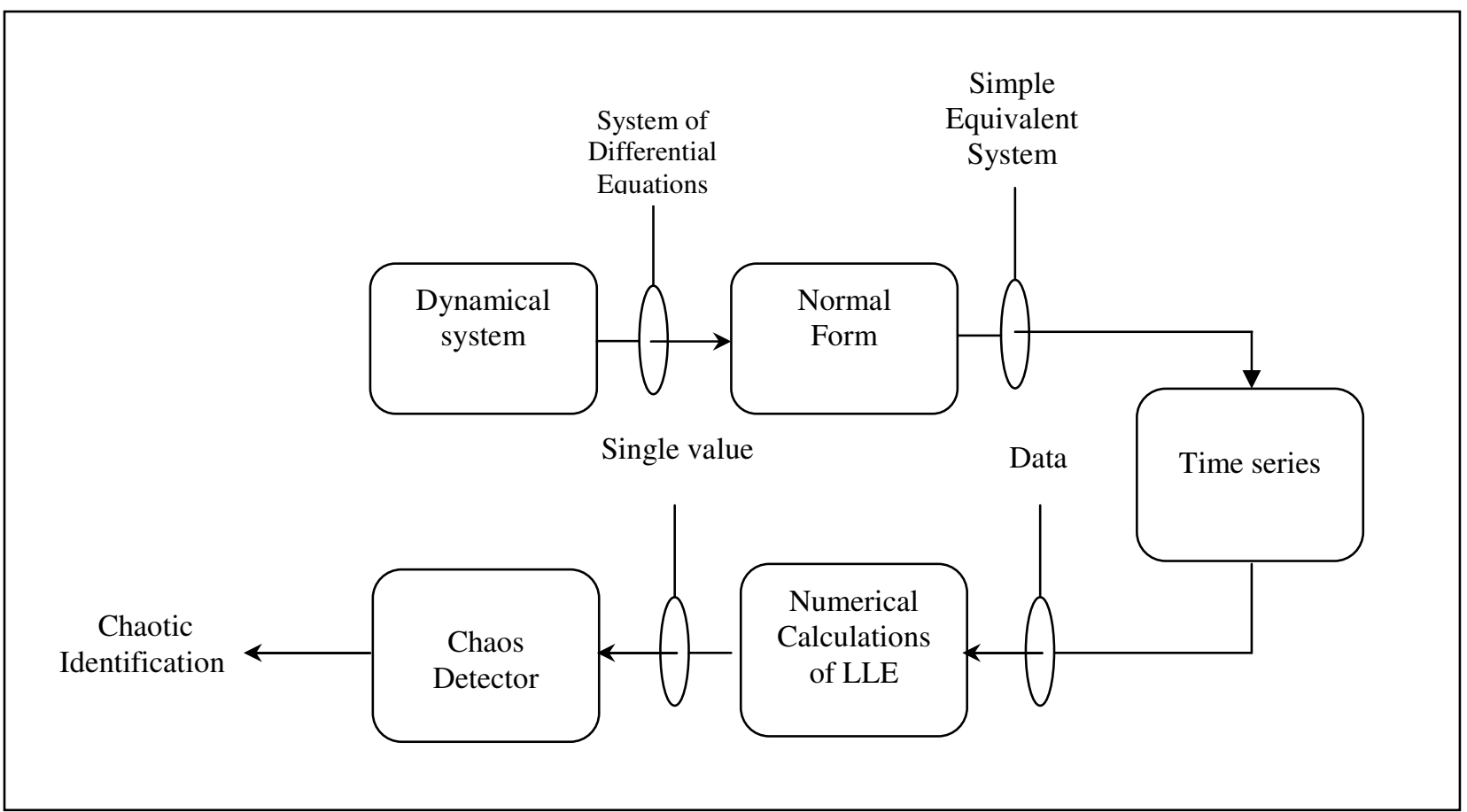

Fig. 1. Block diagram of the proposed algorithm.

- Use the normal form expressions to obtain the time domain solution with the advantage of fast conversion.

- Calculate the LLE from time domain solution.

The outcome of this algorithm is the Lyapunov exponents of the system, the largest of which $\lambda_{i}$ is of special interest in diagnosing dynamical systems, while zero values of $\lambda_{i}$ indicate periodic behavior. Negative values of $\lambda_{i}$ indicate stable fixed points, while positive values of $\lambda_{\mathrm{i}}$ indicate chaotic behavior and presence of chaos [12, $13,14,15]$. The $i^{\text {th }}$ Lyapunov exponent is given by [15]:

$$
\lambda_{i}=\frac{1}{N} \sum_{i=1}^{N} \log \left|\frac{d_{i}}{d_{o}}\right|
$$

where $N$ is the number of solution points obtained by direct integration, $d_{o}$ is the initial separation between two nearby points along the trajectory and $d_{i}$ is the $i^{\text {th }}$ distance between two nearby points on the trajectory. Examples for attractors of a three dimensional system are given in Table.1.

Some examples illustrating the procedure will be introduced in next section. A computer algebra program is designed using package (Maple) to find normal forms. The calculation of the LLE is carried out using an algorithm designed specially in $\mathrm{C}^{++}$ to handle the computation task. 
Table 1. Examples of attractors of a three-dimensional system.

\begin{tabular}{|c|c|c|}
\hline Attractor & $\begin{array}{c}\text { Sign of Lyapunov } \\
\text { exponents }\end{array}$ & Asymptotic dynamics \\
\hline Stable equilibrium & $(-,-,-)$ & Stationary \\
\hline Stable periodic orbit & $(0,-,-)$ & Periodic \\
\hline Attracting torus & $(0,0,-)$ & Quasi-periodic \\
\hline Strange attractor & $(+, 0,-)$ & Chaotic \\
\hline
\end{tabular}

\section{APPLICABLE EXAMPLES}

These examples introduce a comparative study for each of Chua's dynamical system and Coupled quartic oscillators system as follows:

\section{Chua's Dynamical System}

This example introduces a studying Chua's system as follows:

a) Comparison between the analytical solution as normal form and numerical computing one as Runge-Kutta-4 method.

b) Comparison between the calculated LLE using the previously explained methods (standard method and Q-R algorithm) and that calculated based on the proposed method.

\section{Chua's oscillator}

Chua's oscillator is one of the simplest electronic circuits that are capable of producing chaos. It can exhibit a wide array of behavior including a great variety of attractors, bifurcations, and routes to chaos [17].

\section{Chua's system representation}

The Chua's system with cubic nonlinearity is described by [17]:

$$
\begin{aligned}
& \frac{d x}{d t}=\alpha\left(y+c x-x^{3}\right) \\
& \frac{d y}{d t}=x-y+z \\
& \frac{d z}{d t}=-\beta y
\end{aligned}
$$

where $\alpha, c$ and $\beta$ are system parameters. For the set of parameters $(\alpha=10, \beta=16$, and $c=0.143$ ), the system was shown to display Chaos (double scroll attractor) [17]. 
The local Lyapunov exponents can be calculated from the Jacobian matrix at equilibrium point $(\mathrm{x}=\mathrm{y}=\mathrm{z}=0)$ which is given by:

$$
J=\left[\begin{array}{ccc}
-\alpha c & \alpha & 0 \\
1 & -1 & 1 \\
0 & -\beta & 0
\end{array}\right]
$$

The sum of the Lyapunov exponents is given by [14]:

$$
\sum_{i=1}^{3} \lambda_{i}=\lim _{t \rightarrow \infty} \sup \frac{1}{t} \int_{0}^{t} \operatorname{Trace}(J(s)) d s
$$

The result of the calculation gives,

$$
\sum_{i=1}^{3} \lambda_{i}=-\alpha c-1=-2.43
$$

According to [14], this result indicates that the system is dissipative.

\section{Normal form of Chua's system}

For the Chua's system, the $3^{\text {rd }}$ order normal form is given by:

$$
v^{3}=\omega\left(x \frac{\partial}{\partial x}+y \frac{\partial}{\partial y}\right)+\delta\left(y \frac{\partial}{\partial x}-x \frac{\partial}{\partial y}\right)+\sigma z \frac{\partial}{\partial z}
$$

where: $\omega, \theta$ and $\sigma$ are arbitrary constants obtained from the integration of the Ushiki normal form[8]; this results is in agreement with results that obtained in [9].

\section{System behavior using normal form method}

To check the validity of the proposed algorithm, a further illustration of the phase plane and time domain solution using the normal form expressions were illustrated in Fig. 2:

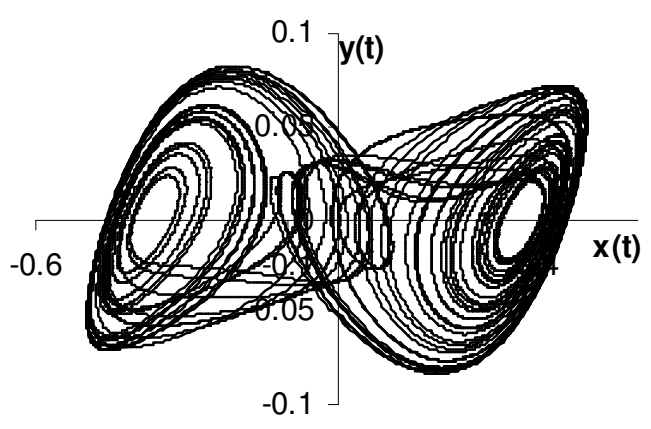

Fig. 2a. The phase plan plot $(x(t), y(t))$ of Chua's system using Normal form method. 


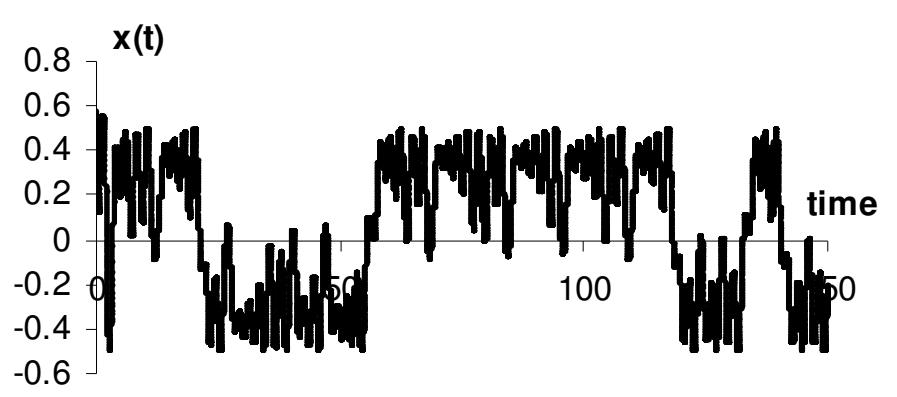

Fig. 2b. The time response $x(t)$ of Chua's system using Normal form method.

\section{System behavior using Runge-Kutta-4 method:}

The numerical solution of the system using the Runge-Kutta-4 method [4] with step size $\mathrm{h}=0.01$ is shown in the phase plane plot and time domain solution of Fig. 3 .

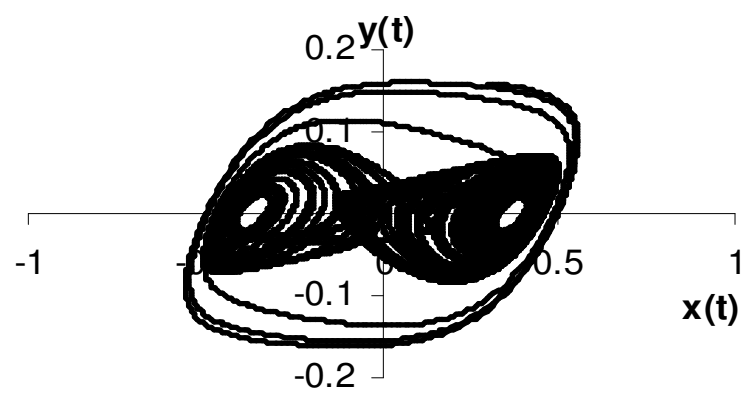

Fig. 3a. The phase plane plot $(x(t), y(t))$ of Chua's system using Runge-Kutta-4 method.

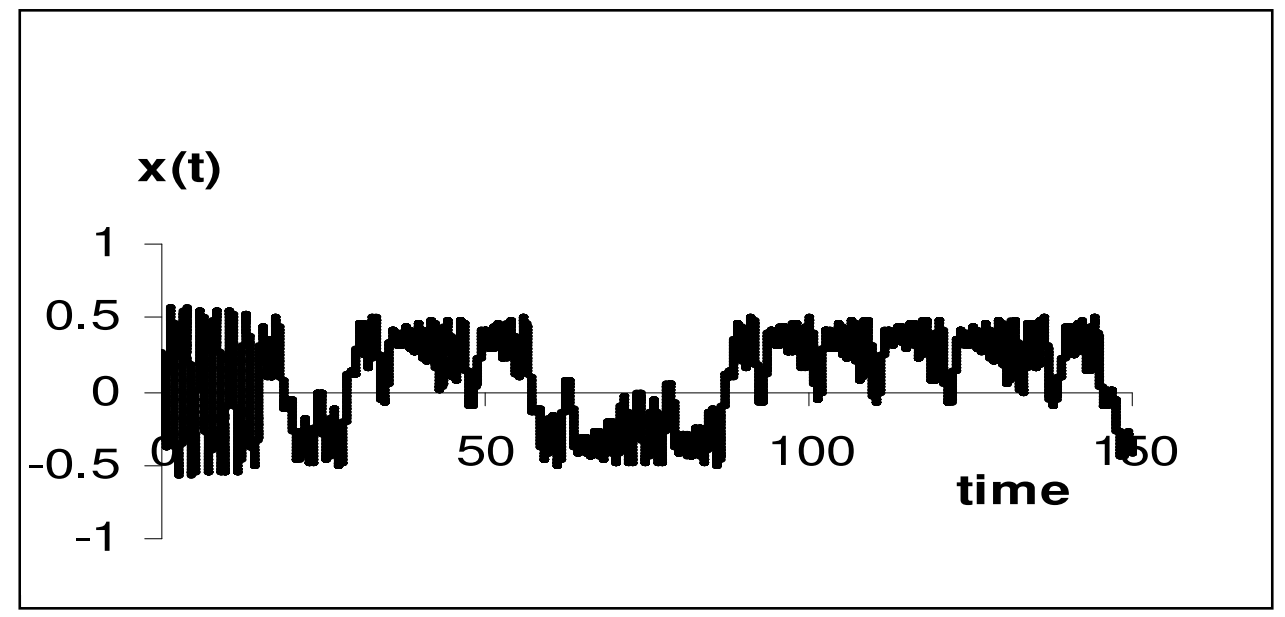

Fig. 3b. The time response $x(t)$ of Chua's system using Runge-Kutta-4 method. 
Figures 2, 3 illustrate that the qualitative behavior of analytical solution is the same as that obtained by the numerical methods with the advantages of fast convergence to steady state behavior. However solutions give the same response, the solution satisfied by the normal form (Fig.2) is free from transient that shown in Fig. 3.

\section{LLE calculations for Chua's system}

Both the standard method and the Q-R algorithm are used to calculate the LLE $(\mathrm{N}=10000$ iterations), respectively. Comparisons between their results and the proposed method are listed in Table 2.

Table.2. Comparisons between existing techniques and the proposed approach.

\begin{tabular}{|c|c|c|c|}
\hline Algorithm & Standard method & Q-R algorithm & $\begin{array}{c}\text { Proposed } \\
\text { approach }\end{array}$ \\
\hline LLE & 0.1332 & 0.1328 & 0.1317 \\
\hline Run time $(\mathrm{sec})$ & 3.09 & 3.99 & 1.84 \\
\hline
\end{tabular}

From this table; the percent of reduction in Run time about $49 \%$ to $59 \%$. The calculations give positive value of the LLE which indicates chaotic behavior (double scroll attractor) and is in agreement with the results of [17].

It is seen from Table 2 that the calculated $\lambda_{1}$ as LLE by using the proposed approach is in good agreement with the existing algorithms with the advantage of minimum run time.

\section{Coupled Quartic Oscillators}

The Coupled quartic oscillators is given by [12]:

$$
\begin{array}{lcc}
\frac{d x}{d t}= & z \\
\frac{d y}{d t}= & w \\
\frac{d z}{d t}= & -\left(4 x^{3}+2 \alpha x y^{2}\right) \\
\frac{d w}{d t}= & -\left(4 y^{3}+2 \alpha y x^{2}\right)
\end{array}
$$

The system is integrable for $\alpha=0,2$ and $\alpha=6$; The sum of Lyapunov exponents is given by:

$$
\sum_{i=1}^{4} \lambda_{i}=0.0
$$

This result indicates that the system is conservative [12] 
Following the same procedure given in the previous example, the results are shown in Fig.4. and Fig. 5.

(i) The solution using Normal form method is shown in Fig.4.

(ii) The solution using Runge- Kutta method is shown in Fig.5.

\section{Normal form of coupled quartic oscillators system}

For Coupled Quartic Oscillator, the $4^{\text {th }}$ order normal form is given by:

$$
v^{4}=(\alpha y) \frac{\partial}{\partial x}+(\beta z) \frac{\partial}{\partial y}+\left(\sigma w z+y^{2}\right) \frac{\partial}{\partial z}+\left(\theta w x^{2}\right) \frac{\partial}{\partial w}
$$

where, $\alpha, \beta, \sigma$ and $\theta$ are the arbitrary constants from the integration of the Ushiki normal form [8].

\section{System behavior using normal form:}

To check the validity of the proposed algorithm a further illustration for the phase plane and time domain solution using the normal form expressions are obtained and presented in Fig.4.

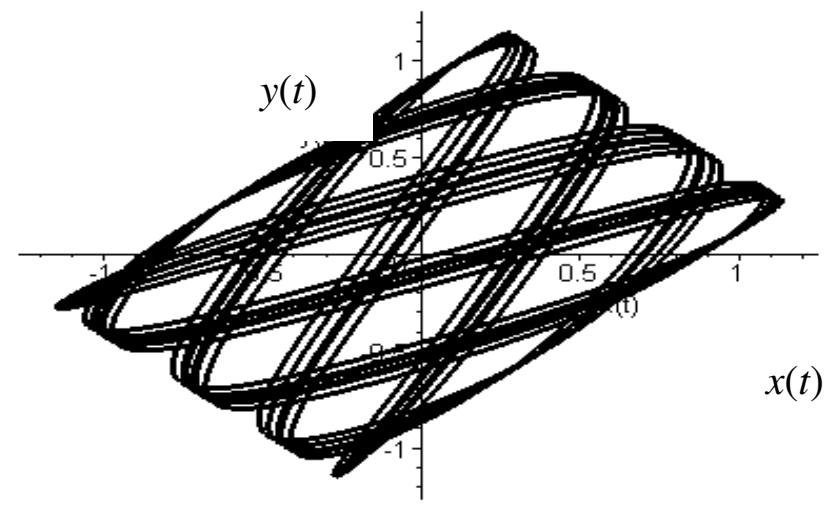

Fig.4a. The phase plan plot $(x(t), y(t))$ of Coupled quartic Oscillators system using Normal form.

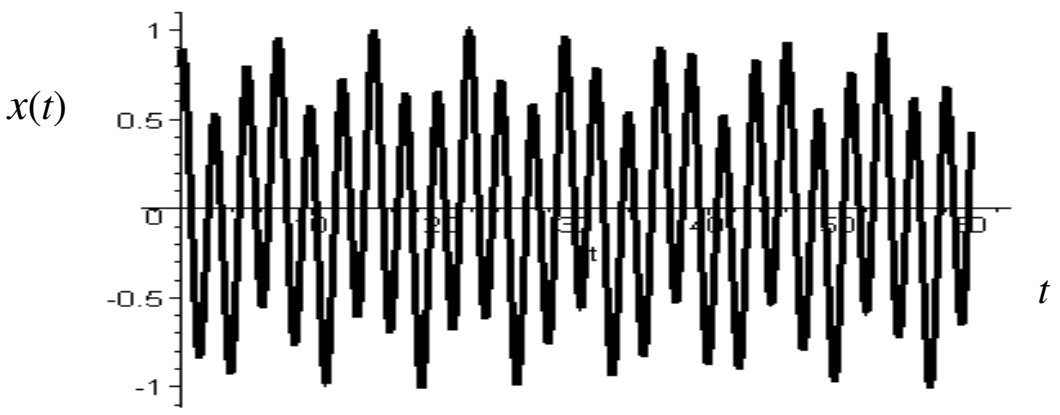

Fig.4b. The time response $x(t)$ of Coupled Quartic Oscillators using Normal form. 


\section{System behavior using Runge-Kutta method:}

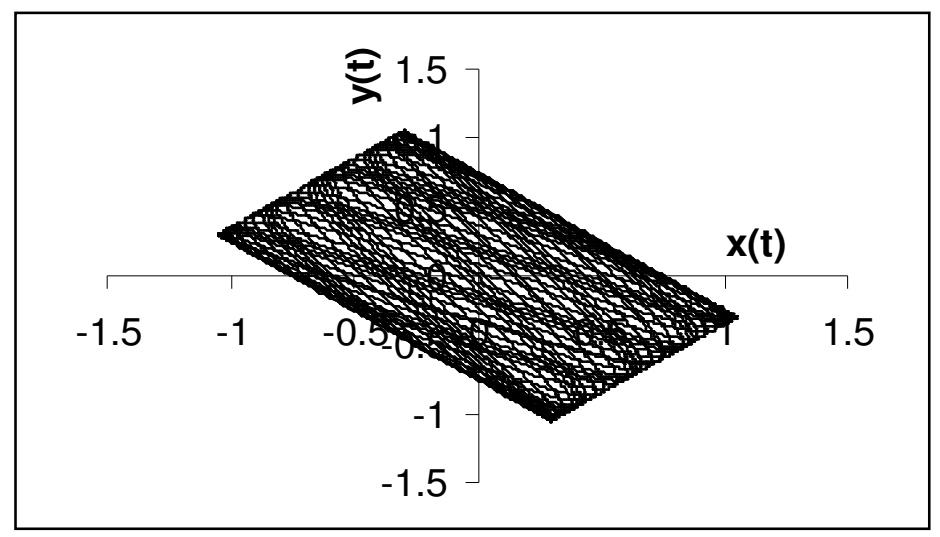

Fig. 5a. The phase plane plot $(x(t), y(t))$ Coupled quartic Oscillators system using Runge-Kutta method , $h=0.01$.

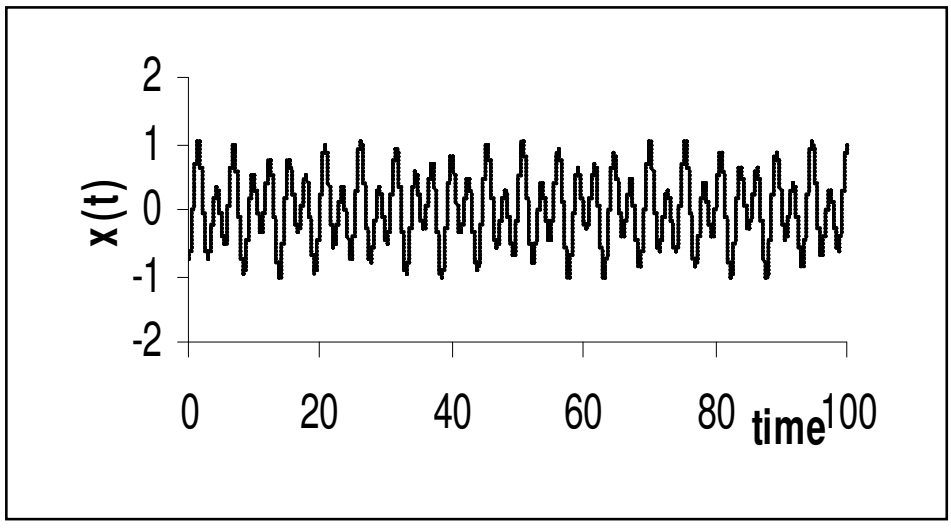

Fig. 5b. The time response $x(t)$ of Coupled quartic Oscillators system using Runge-Kutta method, $\mathrm{h}=0.01$.

Again the qualitative behavior using Normal form method is the same as that obtained by the numerical methods with the advantages of fast convergence to steady state and transient free behavior; and is in agreement with the results in [12].

\section{LLE calculations for coupled quartic oscillators}

The standard method and the $\mathrm{Q}-\mathrm{R}$ algorithm are both used to calculate the largest LLE, $\mathrm{N}=10000$ iterations the results is shown in Table 3.

From this table, the $\%$ reduction in run time about $44 \%$ to $48 \%$. The calculations give positive value of the LLE which indicates chaotic behavior and is in agreement with the results in [12]. 
Table.3. Comparison between existing technique and the proposed approach

\begin{tabular}{|l|c|c|c|}
\hline Algorithm & $\begin{array}{c}\text { Standard } \\
\text { method }\end{array}$ & Q-R algorithm & $\begin{array}{c}\text { Proposed } \\
\text { approach }\end{array}$ \\
\hline LLE & 0.1738 & 0.1793 & 0.1811 \\
\hline Run time (sec) & 4.01 & 4.43 & 1.92 \\
\hline
\end{tabular}

It is seen from Table (3) that the calculated $\lambda_{i}$ by using the proposed approach is in agreement with the existing algorithms with the advantage of minimum run time.

\section{CONCLUSIONS}

- Many numerical techniques are used to investigate the qualitative behavior of the non-linear systems and computing the LLE.

- Most of the used methods have the disadvantages of lack of generality, less accuracy, difficult implementation, and numerical overflows/underflows for higher dimensional systems and give unreal behavior especially in chaotic systems.

- The proposed algorithm that combined the normal form method and the numerical method can provide active solutions to many challenges, avoids most computational errors encountered in other numerical methods, besides it is fast (reduction in run time up to $45 \%$ ) ,transient free, and easily implemented.

- The results of the proposed algorithm indicate it gives some advantages as: fast convergence to steady state solutions, reliable for higher dimensional systems, and low number of iterations required.

\section{REFERENCES}

[1] W. Hirsch and S. Smale, "Differential equations, Dynamical system and linear Algebra," Academic, New York, (1974).

[2] M.Thompson and H.B. Stewart, "Non-linear dynamics and Chaos," Wiley, new york (1986).

[3] J.Guckenheimer and P.J.Holmes, "Non-linear oscillations, Dynamical systems, and Bifurcations of vector fields," NewYork: Springer-verlag, 2nd Edition (1983).

[4] John H. Mathews,"Numerical methods for Mathematics Science, and Engineering," $2^{\text {nd }}$ Edition, Prentice-Hall, Inc. (1992).

[5] J.Della Dora, L.Stolovitch, "Normal forms of differential systems", London Mathematical Society, Lecture note Series (193), CAMBRIDGE UNIVERISITY PRESS.

[6] G. Chen and J. Della Dora, Further reduction of normal forms for dynamical systems, J. Differential Equations 166 (2000) 79-106

[7] S. Ushiki, "Normal form for singularities of vector fields," Japan J.Appl.Math.,Vol.pp-1-37, 1984.

[8] L.O.Chua and H.Kokubu, "Normal forms for non-linear vector fields-part 1: Theory and Algorithm," "IEEE Circuits syst.,vol.35,pp 863-880, (1987). 
[9] Altman, E.J. " Normal form analysis of Chua's circuit with applications for trajectory recognition " Circuits and Systems II: Analog and Digital Signal Processing, IEEE Transactions on (Volume:40, Issue: 10 (1993)

[10] Takens F., Vanderbauwhede A., "Local invariant manifolds and normal forms," Eds Handbook of dynamical systems, vol. 3, North-Holland, (2009).

[11] Michael T. rosestain, James J. collins and carlo J. De Luca, "A practical method for Calculating Largest Lyapunov exponents from small data setes," Neuro muscolar research center and Department of biomedical Engineering, November (1992).

[12] K. Ramasubramanian and M. S. Sriram," A comparative study of computation of Lyapunov spectra with different algorithms," Physica D 139, 72 (2000).

[13] Fridaus E. Udwadia and Hubertus F. von Bremen, "An efficient and stable approach for computation of characteristic exponents for continuous dynamical system," Applied Mathematics and computation, 12, 1219-259 (2001).

[14] Skokos, Ch.: The Lyapunov Characteristic Exponents and Their Computation. Lect. Notes, Phys. 790, 63-135 Springer-Verlag Berlin Heidelberg (2010)

[15] D. Guégan, J. Leroux, "Local Lyapunov Exponents: A New Way to Predict Chaotic Systems, forthcoming in Topics on Chaotic Systems," World Scientific, Eds., (2009) b.

[16] M. Sano and Y. Sawada," Measurement of the Lyapunov spectrum from a chaotic time series," PHYSICAL REVIEW LETTERS, vol35.No10, Sep(1985).

[17] A. Huang, L, Pivka, C. W. Wu and M. Franz, .Chua' s equation with cubic nonlinearity,. Int. J.Bifurcation and Chaos, vol. 6, pp. 2175-2222, 1996. 